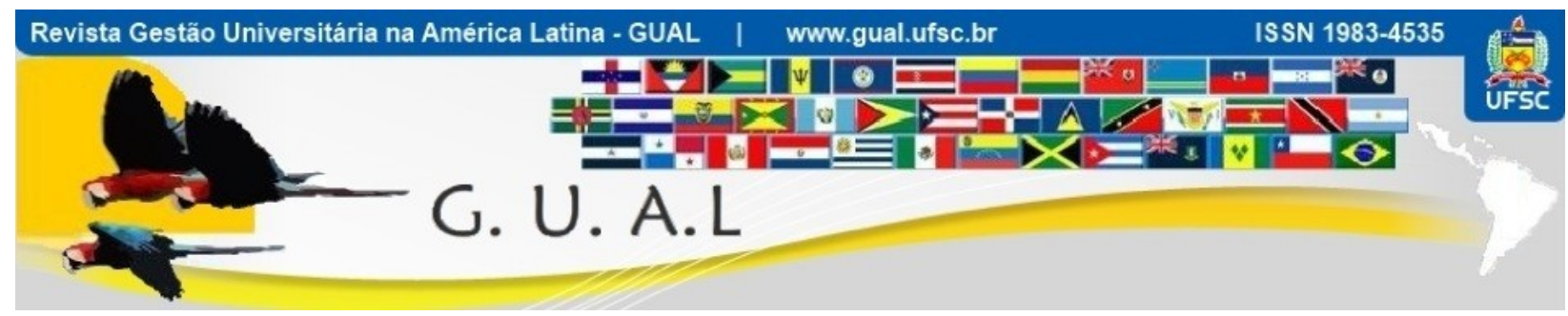

DOI: http://dx.doi.org/10.5007/1983-4535.2013v6n1p212

\title{
AVALIAÇÃO DE CURSOS DE GRADUAÇÃO A PARTIR DA ORIENTAÇÃO PARA O MERCADO
}

\section{EVALUATION OF UNDERGRADUATE COURSES FROM THE MARKET ORIENTATION}

Kenny Basso, Mestre

Faculdade Meridional - IMED

bassokenny@gmail.com

Verner Luis Antoni, Doutor Universidade de Passo Fundo - UPF

antoni@upf.br

Recebido em 26/julho/2012

Aprovado em 19/outubro/2012

Sistema de Avaliação: Double Blind Review

Esta obra está sob uma Licença Creative Commons Atribuição-Uso. 


\title{
RESUMO
}

O artigo é resultado de um estudo desenvolvido para construir e avaliar cursos de graduação a partir de seus stakeholders (profissionais, vestibulandos, alunos e professores). A pesquisa foi dividida em duas etapas, a primeira para explorar atributos relacionados a um curso de graduação, e a segunda para descrever e quantificar esses atributos de acordo com a percepção dos alunos de cursos de graduação em direito, biologia, administração e fisioterapia. Os atributos identificados (corpo docente; estrutura física; currículo) foram relacionados com indicadores de desempenho (satisfação; relação custoXbenefício; relação alunoXprofessor; envolvimento). Ainda, o artigo discute a aplicabilidade do método utilizado e a relação entre os atributos e os indicadores de desempenho na formulação de um curso de graduação orientado para o mercado.

Palavras-chave: Instituições de ensino superior. Orientação para o mercado. Indicadores de desempenho.

\begin{abstract}
The article is the result of a study designed to build and evaluate undergraduate courses from its stakeholders (market professionals, school students, undergraduate students and teachers). The survey was divided into two stages, the first to explore related attributes to an undergraduate program, and the second to describe and quantify these attributes according to the students' perception of undergraduate courses in law, biology, management and physical therapy. The attributes identified (faculty; physical structure; curriculum) were related to performance indicators (satisfaction; relation between cost and benefits; relation between students and faculty; involvement). Still, the article discusses the applicability of the method used and the relationship between the attributes and performance indicators in the formulation of a market-oriented undergraduate course.
\end{abstract}

Keywords: Institutions of higher education. Market orientation. Performance indicators. 


\section{INTRODUÇÃO}

De acordo com dados do INEP (2012) existem atualmente 2.378 Instituições de Ensino Superior, sendo 2.100 privadas e 278 públicas. No estado do Rio Grande do Sul atuam 115 Instituições de Ensino Superior (IES), sendo 105 privadas e 10 públicas. Nesse mercado o estado é responsável pela oferta de cerca de 130.000 vagas entre instituições públicas e privadas.

Neste ambiente competitivo, surge a discussão da necessidade e importância de direcionar as ações das instituições de forma mais orientada ao mercado tendo em vista a competitividade existente nesse mercado (NICOLAO; ESPARTEL, 2004), para tanto, esse tipo de instituição têm de rever suas posturas mercadológicas, bem como adaptar suas formas de gestão, ensino, pesquisa e extensão para assim poderem estabelecer vínculos e contatos mais diretos com o mercado (SOUZA et al., 2004).

Como indicam os números do INEP (2012) a competição ocorre na maioria dos cursos de graduação, onde há uma crescente oferta de vagas nesse setor, o que para Antoni (2004) implica na busca por uma maior orientação para o mercado, pois os clientes devem ter seus desejos e suas expectativas atendidas frente ao serviço que irão contratar. Essa orientação para o mercado aplicada a instituições de ensino superior, segundo Antoni, Damacena e Lezana (2004) é necessária para a sustentabilidade da organização através de uma busca de vantagens competitivas sustentáveis frente a seus concorrentes.

A necessidade de um melhor direcionamento das ações de instituições de ensino superior também foi constatada por Faleiro (2001) quando afirma que um processo organizado de orientação para o mercado torna as instituições capazes de efetuarem processos contínuos de inovação e aprendizagem através do entendimento de seus stakeholders, em prol de manter a instituição atuante.

Nesse contexto o artigo apresenta a metodologia e os resultados de um estudo desenvolvido em uma Instituição de Ensino Superior, com 20.000 mil alunos e 48 cursos de graduação que está localizada ao Norte do estado do Rio Grande do Sul. O estudo foi desenvolvido junto aos cursos de direito, biologia, administração e fisioterapia.

Para uma melhor compreensão do artigo, num primeiro momento é apresentado o aporte teórico referente a orientação para o mercado e perfomance e a utilização da orientação em IES. Na seqüência é detalhada a metodologia utilizada e os resultados da pesquisa desenvolvida, por fim são discutidas as conclusões e implicações do estudo. 


\section{REFERENCIAL TEÓRICO}

\subsection{ORIENTAÇÃO PARA O MERCADO E PERFORMANCE}

A orientação para o mercado, segundo Kohli e Jaworski (1990), reside na capacidade organizacional de gerar, disseminar e responder a inteligência, voltando as ações para as necessidades atuais e futuras dos consumidores. Os mesmos autores (1990) discutem a dificuldade de transpor o conceito de marketing para a prática empresarial e, por conseqüência, alcançar a orientação para o mercado, uma vez que uma organização orientada para o mercado é aquela que implementa e que age de acordo com o conceito de marketing. Jaworski e Kohli (1993) identificaram que quanto mais alta a orientação para o mercado, maior será a performance empresarial, independentemente da turbulência do mercado, da intensidade da competição e da turbulência da tecnologia presentes no ambiente em que a empresa atua, ou seja, independente da atuação de fatores exógenos.

As empresas orientadas para o mercado, segundo Day (1994), possuem performance superior, pois geram e sustentam capacidades distintas que permitem satisfazer seus clientes, oferecendo valor superior ao ofertado pelos seus concorrentes. Para tanto, devem enfatizar o papel da cultura, da obtenção e utilização da informação e da coordenação interfuncional. $O$ desenvolvimento dessas capacidades foi foco do estudo de Day (1994, p. 38), que as define como ligações complexas dos recursos e conhecimento acumulado, exercido através do processo organizacional, que permite a empresa coordenar atividades e usar seus recursos. Continuando no mesmo raciocínio, a orientação para o mercado representa uma habilidade superior no entendimento da satisfação dos clientes (DAY, 1994, p. 38).

Day (2001) afirma que orientar-se para o mercado é ter disciplina para fazer opções estratégicas saudáveis e implantá-las de forma coerente e completa, não sendo "tudo para todos".

Já, Narver e Slater (1990), definem a orientação para mercado como uma cultura organizacional que efetivamente e eficientemente cria comportamentos necessários para a geração de valor superior para o consumidor e desta forma proporciona um melhor desempenho organizacional. No entanto, os mesmos autores (1990) afirmam que a criação de valor para os compradores não é função única de marketing, pois todas as áreas da empresa devem, como uma grande orquestra, contribuir para isso de forma sinérgica.

Nesse sentido as organizações ao se orientarem para o mercado obtêm performance superior e vantagens competitivas sustentáveis, o que para Antoni (2004) vêm se tornando 
cada vez mais importante para ampliar a capacidade competitiva das Instituições de Ensino Superior.

\subsection{ORIENTAÇÃO PARA O MERCADO E MARKETING EM IES}

A competição crescente no setor de ensino brasileiro não é algo novo, porém é um fenômeno que exige atenção dos gestores de IES (ANTONI et al., 2004). De acordo com Faleiro (2001), é vital para as instituições de ensino superior que almejam permanecer no mercado tomar ciência dos desejos e preferências dos seus clientes atuais e potenciais. Esta competitividade é uma implicação da globalização, tão discutida no meio empresarial e que afeta também as instituições de ensino superior, que segundo Odin e Manicas (2004) não competem apenas pela demanda de educação superior, mas também por recursos para a pesquisa. Ainda, Eboli (1999) trata que a educação está conectada com a modernidade e a competitividade, uma vez que atua no desenvolvimento dos indivíduos e por conseqüência indireta na sociedade e nas organizações do meio.

As instituições de ensino superior como unidades mercadológicas constituídas e atuantes passam a tratar seus estudantes como consumidores, em uma perspectiva como a descrita por Eagle e Brennan (2007), na qual o estudante é encarado como consumidor participante, pois atua na construção do serviço e também faz parte dos processos inerentes ao sucesso ou insucesso do mesmo no tempo (ZEITHAML; BITNER, 1996; KELLEY et al., 1990; SIMPSON; SIGUAW, 2000). Por outro lado, Maringe (2006) afirma que os estudantes estão assumindo uma postura mais consumista quando da tomada de decisão relacionada à escolha de uma IES, contudo cabe aos gestores de instituições de ensino superior atentarem não só os alunos-consumidores, mas também para os diversos stakeholders que inferem em seus processos de alguma forma (KOTLER; FOX, 1994; FROEMMING, 2001; NAUDÉ; IVY, 1999). Kotler e Fox (1994), constatando os conflitos de interesses dos diversos públicos, consideram que um público torna-se mercado quando a instituição decide que deseja atrair certos recursos (participação, colaboração, doações entre outras formas de recursos) desse público, através de um grupo de benefícios ofertados em troca. Para ser bem-sucedida, ela dedicará esforços para aprender mais sobre este público e planejará uma oferta para engajá-la prontamente no mercado.

Ainda Kotler e Fox (1994) evidenciam diversos stakeholders possíveis para uma instituição de ensino superior, dentre os quais estudantes potenciais, alunos matriculados e a comunidade empresarial. Em observância a Tachizawa e Andrade (2001), uma IES deve ter 
uma constante melhora em seus processos educacionais, despendendo cada vez mais atenção a expectativas e desejos mais específicos e exigentes por parte dos seus stakeholders, o que em um ambiente competitivo deve ser feito pelo marketing bem estruturado da IES.

Neste ambiente competitivo, Venkatesh (2001) afirma que o marketing em instituições de ensino superior começou a ser trabalhado como ferramenta gerencial uma vez que passaram a ser encontradas missões e objetivos desconexos com a realidade mercadológica vivida, necessidade de sustentação para determinadas ações e departamentos, nascimento de programas e cursos similares, falta de diferenciações entre instituições concorrentes, redução de tempo por parte dos estudantes, necessidade de otimização dos recursos monetários e o aumento do custo da instrução.

Antoni (2004) remete a importância da adoção de práticas de orientação ao mercado pelas instituições de ensino superior, dando atenção aos diversos stakeholders através do entendimento dos perfis e das percepções destes quanto a um curso de ensino superior adequado as suas expectativas. Em um ambiente repleto de mudanças e processos que as impõe, Kotler e Fox (1994) afirmam ser necessário atentar para os clientes e para o mercado não só em momentos críticos de escassez, mas sim durante toda a existência da instituição de ensino, tendo em vista a perenidade dos serviços no mercado.

O marketing em IES segundo Kotler e Fox (1994), ocorre quando os gestores passam a analisar seus ambientes, mercados, concorrentes, avaliar forças e fraquezas e desenvolvem um sentido claro de missão, mercados-alvo e posicionamento de mercado.

Os processos de marketing das IES devem procurar entender os atributos valorizados pelos estudantes atuais quando da decisão de ingresso, que por sua vez, diferem dos atributos valorizados antigamente (ABRAHAMSON, 2000). Nisto, vários estudos já foram feitos visando entender tais atributos (SPIES, 1978; SEVIER, 1993; GALOTTI; MARK, 1994; HU; HOSSLER, 2000). Entender os atributos é recorrer ao entendimento das percepções da idealização de um curso, de uma instituição, e, por conseguinte, entender as expectativas e desejos que devem ser atendidos pelo serviço a ser prestado. Determinar quais os atributos de um serviço que são valorizados pelos consumidores é essencial para uma instituição direcionar seus processos para satisfação dos seus stakeholders, no entanto cabe a instituição ter conhecimento de quais são esses atributos e quão importante o são para determinados stakeholders (GUSTAFSSON; JOHNSON, 2004).

Os atributos importantes são mutáveis durante o tempo e ciclo de vida do serviço (NILSSON-WITELL; FUNDIN, 2005), deste modo cabe as instituições adotarem um 
processo contínuo de monitoramento dos atributos e das mudanças que ocorrem ou poderão ocorrer, traçando assim estratégias mais direcionadas ao mercado para que o serviço torne-se mais duradouro, não sucumbindo a mudanças inesperadas.

Durvasula, Lysonski e Mehta (2000) citam que uma das atividades do marketing é manter o cliente satisfeito durante o maior período de tempo possível, e que tal patamar se atinge quando o serviço entregue está de acordo ou é superior ao esperado pelo cliente. De forma inversa, prover um serviço inadequado às expectativas dos clientes irá causar insatisfações e descontentamentos nestes, o que pode vir a prejudicar a sustentabilidade do serviço educacional no tempo.

A transmissão do conhecimento em educação superior caracteriza-se essencialmente como um serviço, que segundo Lovelock e Wright (2003, p.5) é um ato ou desempenho oferecido por uma parte à outra. Da mesma forma Siqueira e Carvalho (2006) consideram o ensino como prestação de serviço uma vez que desenvolve atividades essencialmente de serviço, tal como a transmissão de conhecimentos através de aulas e palestras ao mesmo tempo em que fornece serviços de apoio como biblioteca e secretaria.

Leite et al. (2006) citam que as expectativas dos clientes devem ser atendidas e para tanto, há de se distinguir as reais percepções dos clientes das percepções dos gerentes ofertantes do serviço. Os mesmos autores ainda afirmam que as expectativas dos clientes podem variar quanto a níveis e, em diversas vezes, são diferentes das expectativas que o serviço se propõe a suprir.

Uma distinção acerca da intenção de compra do consumidor e do efetivo serviço envolve questionamentos em torno de que interesse, objetivo e necessidade são atendidos através da aquisição do serviço (JAAKKOLA, 2007). Através de questionamentos busca-se entender os processos que norteiam as decisões dos consumidores. Para responder a esses questionamentos o marketing utiliza-se da pesquisa de campo para analisar quais os itens devem ser trabalhados e de que forma devem ser desenvolvidos para que atinjam os objetivos esperados com sucesso, a partir das percepções dos stakeholders presentes no ensino superior.

\section{MÉTODO}

A pesquisa desenvolvida ocorreu em uma IES do Norte do estado do Rio Grande do Sul, a qual conta com 48 cursos de graduação, tendo atualmente uma abrangência em torno de 100 municípios, e 20.000 alunos. 
Foram utilizados 4 cursos da Instituição para o desenvolvimento da pesquisa sendo eles: direito; biologia; administração e fisioterapia. Os cursos pesquisados foram selecionados pela própria instituição de acordo com julgamentos de sua própria necessidade interna de informações.

Para alcançar o objetivo proposto o estudo foi desenvolvido em duas etapas distintas, porém complementares, a primeira exploratória-qualitativa, enquanto a segunda foi descritivaquantitativa. As duas etapas foram feitas em corte-transversal, conforme sugere Malhotra (2006) para este tipo de pesquisa.

\subsection{ETAPA EXPLORATÓRIA-QUALITATIVA}

A etapa teve como objetivo principal gerar atributos que devem estar contidos em um curso de graduação a partir de uma perspectiva do mercado, quando foram consideradas as percepções de alunos, vestibulandos, profissionais do mercado e professores.

Experts do setor (professores de marketing, pesquisadores em ensino superior e gestores de IES) foram selecionados segundo critério de conveniência e representatividade na área, foram contatados, para formular juntamente com o referencial teórico apresentado no artigo, os roteiros semi-estruturados que posteriormente foram utilizados na realização dos grupos de foco e entrevistas de profundidade.

A população desta etapa foi composta por quatro categorias de stakeholders relacionados aos cursos de graduação em direito, biologia, administração e fisioterapia: (1) alunos regularmente matriculados no curso; (2) vestibulandos com intenção de ingressar no curso; (3) profissionais atuantes no mercado e ligados ao curso em análise; (4) professores do curso.

A amostragem para esta etapa foi não-probabilística, enquadrada, de acordo com Malhotra (2006) em uma amostragem por conveniência, onde os entrevistados foram selecionados de acordo com a conveniência dos entrevistadores.

A coleta dos dados foi feita através de duas técnicas (MALHOTRA, 2006): grupos de foco e entrevistas em profundidade. Os grupos de foco foram utilizados com os públicos de alunos atuais e vestibulandos com interesse no curso, sendo que a quantidade realizada por curso ficou distribuída da seguinte forma:

- Direito: 9 grupos focais com alunos e 1 com vestibulandos;

- Biologia: 2 grupos focais com alunos; 
- Administração: 9 grupos focais com alunos e 1 com vestibulandos.

Para o curso de fisioterapia tanto alunos quanto vestibulandos foram abordados com entrevistas em profundidade. A mesma abordagem foi utilizada com os alunos de biologia. Também foram realizadas entrevistas em profundidade junto aos profissionais de mercado e professores dos cursos. A distribuição das entrevistas em profundidade é apresentada a seguir:

- Direito: 10 entrevistas em profundidade com professores e 20 com profissionais de mercado;

- Biologia: 6 entrevistas em profundidade com professores, 8 com profissionais de mercado e 7 com vestibulandos;

- Administração: 10 entrevistas em profundidade com professores e 20 com profissionais de mercado;

- Fisioterapia: 9 entrevistas em profundidade com professores, 21 com profissionais de mercado, 11 com vestibulandos e 30 com alunos.

Convém destacar que foram utilizadas duas técnicas de coleta em função da distribuição geográfica dos stakeholders e também da disponibilidade de tempo dos mesmos.

Os grupos focais e entrevistas em profundidade foram transcritos e analisados, através da técnica de análise de conteúdo, com codificação categórica (BARDIN, 2004; CAPPELLE et al., 2003). Convém ressaltar que dois pesquisadores participaram da análise e que cada um fez a sua análise e posteriormente estas foram comparadas visando identificar semelhanças e divergências, onde ajustes de categorias foram feitos.

\subsection{ETAPA DESCRITIVA-QUANTITATIVA}

Esta segunda etapa seguiu o método survey de natureza descritiva-quantitativa (MALHOTRA, 2006), tendo como objetivo descrever a importância dos atributos gerados na etapa qualitativa e também permitiu verificar o impacto destes atributos em variáveis de performance do curso de graduação.

A população desta etapa foi composta pelos alunos regularmente matriculados nos cursos de direito, biologia, administração e fisioterapia da IES em análise. A amostragem foi probabilística e aleatória por grupos, que segundo Malhotra (2006) pode ser feita quando é possível distribuir a população em grupos e, aleatoriamente, selecionar os grupos que irão ser 
os respondentes da pesquisa. Desta forma, as turmas de alunos constituíram-se nos grupos, que por sua vez foram aleatoriamente distribuídos para participarem da pesquisa.

Os tamanhos das amostras foram calculados para cada curso, com vista a inferir uma margem de erro de 5\% e um coeficiente de segurança de $95 \%$ para a pesquisa (MALHOTRA, 2006). O Quadro 1 descreve a população e amostra.

$\mathrm{Na}$ amostra total, $60 \%$ dos respondentes são homens, a média de idade ficou entre $21 \mathrm{e}$ 25 anos, com rendimentos mensais variando entre $\mathrm{R} \$ 927,00$ a $\mathrm{R} \$ 1.669,00$. A maioria dos respondentes $(77 \%)$ é solteira.

\begin{tabular}{|c|c|c|}
\hline Público & População & Amostra \\
\hline Direito & 2.314 & 358 \\
\hline Biologia & 259 & 156 \\
\hline Administração & 1.312 & 299 \\
\hline Fisioterapia & 275 & 161 \\
\hline Total & 4.160 & 974 \\
\hline
\end{tabular}

Quadro 1 Descrição da população e amostra

Fonte: Dados da Pesquisa.

O instrumento utilizado para a coleta dos dados foi elaborado com base nos indicadores que foram identificados na etapa exploratória-qualitativa, contendo questões do tipo fechada-múltipla onde se procurava mensurar a importância dos atributos e questões escalares nas quais foi utilizada uma escala Likert de 5 pontos (discordo totalmente a concordo totalmente).

Os atributos (corpo docente; estrutura física; currículo) e as medidas de performance do curso (satisfação; custo x benefício; relação aluno x professor; envolvimento), foram definidos na etapa anterior do estudo.

O instrumento teve sua validação de conteúdo feita por um comitê de 3 juízes e práticos (MALHOTRA, 2006). Foi realizado também um pré-teste para cada curso analisado, com uma coleta de 30 casos em cada situação, procurando verificar as adequações do instrumento aos respondentes, o tempo de preenchimento e a forma de abordagem para o preenchimento.

A coleta dos dados foi feita em sala de aula, contando com a colaboração dos professores. Após coletados, os dados foram tratados no Software SPSS®. Para tanto, foi analisada a qualidade do banco de dados; os missing values, que foram substituídos pela média em cada variável, sendo que não foram encontrados valores maiores que 5\% para cada variável; os outliers foram identificados por estatísticas univariadas (teste z) e multivariadas (distância $\mathrm{D}^{2}$ de Mahalanobis). Após retirados da análise, a normalidade dos dados foi 
comprovada por meio de cálculos de assimetria e curtose, enquanto a multicolinearidade foi verificada por meio de cálculos de correlação bivariada e pelo cálculo de tolerância, não sendo encontrado nenhum caso. Por fim, a homocedasticidade dos dados foi verificada por meio do teste de Levene (HAIR et al., 2005). Após os testes, a base de dados foi considerada adequada para os procedimentos de análise multivariada (HAIR et al., 2005).

Para a análise dos dados foram adotados processos de estatística descritiva, com testes de diferenças de médias. Também foram utilizadas técnicas multivariadas como a análise discriminante e a regressão múltipla. Na pesquisa para aceitação das relações, a significância adotada ( $p$-value) foi inferior a 0,05 .

\section{RESULTADOS DA ANÁLISE DOS DADOS}

A análise dos dados revelou que os principais atributos valorizados pelos acadêmicos são o corpo docente, a estrutura física e o currículo. A tabela 1, mostra as diferentes freqüências obtidas para cada grupo em análise, que por sua vez são estatisticamente distintas entre grupos, de acordo com o cálculo do qui-quadrado $\left(\chi^{2}=439,296 \mathrm{gl}=33 ; \mathrm{p}=0,000\right)$.

Tabela 1 Atributos mais valorizados na escolha de curso de graduação

\begin{tabular}{c|c|c|c|c|c}
\hline \multirow{2}{*}{ Atributos Valorizados } & \multicolumn{3}{|c|}{ Curso } & \multirow{2}{*}{ Total } \\
\cline { 2 - 5 } & Direito & Biologia & Administração & Fisioterapia & \\
\hline Corpo Docente & $65,4 \%$ & $39,1 \%$ & $45,8 \%$ & $12,4 \%$ & $46,4 \%$ \\
\hline Estrutura Física & $3,4 \%$ & $5,8 \%$ & $4,7 \%$ & $27,3 \%$ & $8,1 \%$ \\
\hline Currículo & $7,8 \%$ & $12,2 \%$ & $7,4 \%$ & $9,3 \%$ & $8,6 \%$ \\
\hline Localização & $2,8 \%$ & $0,6 \%$ & $3,3 \%$ & $3,7 \%$ & $2,8 \%$ \\
\hline Outros & $20,6 \%$ & $42,3 \%$ & $38,8 \%$ & $47,3 \%$ & $4,2 \%$ \\
\hline Total & $100,0 \%$ & $100,0 \%$ & $100,0 \%$ & $100,0 \%$ & $100,0 \%$ \\
\hline
\end{tabular}

Fonte: Dados da pesquisa.

Com base nos três principais atributos encontrados (corpo docente; estrutura física; currículo), a tabela 2 mostra a diferença das médias destes atributos para os quatro cursos analisados. Considerando a importância destes três atributos na elaboração da oferta destes cursos de graduação, foi identificado que as médias dos três atributos têm diferenças significativas entre os quatro cursos analisados, como mostra a tabela 2, onde valores do teste de Wilks'Lambda inferiores a 1 indicam que as médias do referido atributo para os cursos são distintas entre si, uma vez que valores igual ou próximos a 1 indicam semelhança ou igualdade entre as médias. 
Tabela 2 Diferença das médias entre grupos para os três atributos mais valorizados

\begin{tabular}{c|c|c|c|c|c}
\hline Atributos & Wilks' Lambda & F & gl1 & gl2 & Sig. \\
\hline Corpo Docente & 0,700 & 138,557 & 3 & 970 & 0,000 \\
\hline Estrutura Física & 0,802 & 79,994 & 3 & 970 & 0,000 \\
\hline Currículo & 0,552 & 262,258 & 3 & 970 & 0,000 \\
\hline
\end{tabular}

Fonte: Dados da pesquisa.

Para identificar os impactos destes atributos em variáveis de resultado para a IES, foram feitas regressões múltiplas, tendo os atributos como variáveis independentes e a variável de performance (satisfação; custo $\mathrm{x}$ benefício; relação aluno $\mathrm{x}$ professor; envolvimento) como dependente. A seguir são apresentados os resultados por cursos estudados, sendo que cada tabela mostra as regressões estabelecidas, seus respectivos coeficientes de regressão $(\beta)$ e coeficientes de significância, bem como os coeficientes de explicação $\left(\mathrm{R}^{2}\right)$ para cada construto examinado.

No curso de direito (tabela 3), a satisfação foi o indicador de resultado com maior explicação pelos indicadores preditores (32\%) tendo o corpo docente como o principal atributo de impacto, apesar da estrutura física e o currículo também terem mostrado relações significativas com a satisfação. A percepção de custo $\mathrm{x}$ benefício, embora com um baixo nível de explicação (6\%), teve um coeficiente de regressão negativo quando o corpo docente era a variável independente, o que demonstra a percepção de que qualidade está associada a custos. Na relação aluno x professor, o corpo docente e o currículo mostraram relações significativas, enquanto apenas o corpo docente teve impacto significativo no envolvimento do aluno com o curso.

Tabela 3 Regressão múltipla entre atributos e indicadores de resultado - curso de direito

\begin{tabular}{|c|c|c|c|}
\hline Regressão & Coeficientes $\beta$ & Sig. & $\mathbf{R}^{2}$ \\
\hline Satisfação $\leftarrow$ Corpo Docente & 0,338 & $0,000^{*}$ & \multirow{3}{*}{0,320} \\
\hline Satisfação $\longleftarrow$ Estrutura Física & 0,152 & $0,002 * *$ & \\
\hline Satisfação $\leftarrow$ Currículo & 0,226 & $0,000^{*}$ & \\
\hline Custo X Benefício $\leftarrow$ Corpo Docente & $-0,187$ & $0,002 * *$ & \multirow{3}{*}{0,068} \\
\hline Custo X Benefício $\longleftarrow$ Estrutura Física & $-0,091$ & 0,110 & \\
\hline Custo X Benefício $\longleftarrow$ Currículo & $-0,044$ & 0,456 & \\
\hline Relação aluno X professor $\leftarrow$ Corpo Docente & 0,285 & $0,000 *$ & \multirow{3}{*}{0,224} \\
\hline Relação aluno X professor $\leftarrow$ Estrutura Física & 0,077 & 0,137 & \\
\hline Relação aluno X professor $\leftarrow$ Currículo & 0,224 & $0,000^{*}$ & \\
\hline Envolvimento $\leftarrow$ Corpo Docente & 0,148 & $0,014 * *$ & \multirow{3}{*}{0,059} \\
\hline Envolvimento $\leftarrow$ Estrutura Física & 0,044 & 0,440 & \\
\hline Envolvimento $\leftarrow$ Currículo & 0,109 & 0,066 & \\
\hline
\end{tabular}

$\mathrm{Na}$ análise do curso de biologia (tabela 4), a satisfação do aluno está ligada à estrutura física disponível e ao currículo, uma vez que na relação do corpo docente esta variável não foi significativa. A relação entre o custo e o benefício percebido não obteve nenhuma relação 
significativa com os atributos, e, por conseguinte, um baixíssimo coeficiente de explicação $(0,9 \%)$. A relação entre aluno e professor, por sua vez, sofre impacto do currículo do curso $(\beta=0,234)$. O envolvimento do aluno com o curso, no grupo de biologia analisado, sofre impacto significativo da estrutura física.

Tabela 4 Regressão múltipla entre atributos e indicadores de resultado - curso de biologia

\begin{tabular}{|c|c|c|c|}
\hline Regressão & Coeficientes $\beta$ & Sig. & $\mathbf{R}^{\mathbf{2}}$ \\
\hline Satisfação $\longleftarrow$ Corpo Docente & 0,101 & 0,199 & \multirow{3}{*}{0,189} \\
\hline Satisfação $\leftarrow$ Estrutura Física & 0,313 & $0,000 *$ & \\
\hline Satisfação $\leftarrow$ Currículo & 0,175 & $0,032 * *$ & \\
\hline Custo X Benefício $\leftarrow$ Corpo Docente & $-0,065$ & 0,456 & \multirow{3}{*}{0,009} \\
\hline Custo X Benefício $\leftarrow$ Estrutura Física & 0,001 & 0,994 & \\
\hline Custo X Benefício $\leftarrow$ Currículo & $-0,050$ & 0,579 & \\
\hline Relação aluno X professor $\leftarrow$ Corpo Docente & 0,077 & 0,357 & \multirow{3}{*}{0,072} \\
\hline Relação aluno X professor $\leftarrow$ Estrutura Física & $-0,013$ & 0,873 & \\
\hline Relação aluno X professor $\leftarrow$ Currículo & 0,234 & $0,007 * *$ & \\
\hline Envolvimento $\leftarrow$ Corpo Docente & 0,097 & 0,242 & \multirow{3}{*}{0,095} \\
\hline Envolvimento $\leftarrow$ Estrutura Física & 0,213 & $0,009 * *$ & \\
\hline Envolvimento $\leftarrow$ Currículo & 0,115 & 0,178 & \\
\hline
\end{tabular}

$* \mathrm{p} \leq 0,001 * * \mathrm{p} \leq 0,05$

Fonte: Dados da pesquisa.

No curso de administração (tabela 5), os resultados indicam que a predição da satisfação (29\%) e da relação entre aluno e professor (29\%) é superior a predição da relação custo $\mathrm{x}$ benefício (4\%) e do envolvimento do aluno (11\%), pelos três atributos em análise, sendo assim é possível que para este curso os atributos possuam maior relacionamento com a satisfação e a relação entre aluno-professor. A satisfação teve uma relação significativa com os três atributos, mas o coeficiente de regressão foi mais elevado para a relação entre o corpo docente e a satisfação $(\beta=0,400)$. Já a variável custo $\mathrm{x}$ benefício obteve relação significativa apenas com o corpo docente, onde a magnitude mostrou-se inversa, isto é a relação entre os dois construtos é negativa.

Tabela 5 Regressão múltipla entre atributos e indicadores de resultado - curso de administração

\begin{tabular}{|c|c|c|c|}
\hline Regressão & Coeficientes $\beta$ & Sig. & $\mathbf{R}^{2}$ \\
\hline Satisfação $\leftarrow$ Corpo Docente & 0,400 & $0,000^{*}$ & \multirow{3}{*}{0,291} \\
\hline Satisfação $\leftarrow$ Estrutura Física & 0,110 & $0,043 * *$ & \\
\hline Satisfação $\leftarrow$ Currículo & 0,148 & $0,012 * *$ & \\
\hline Custo X Benefício $\leftarrow$ Corpo Docente & $-0,154$ & $0,020 * *$ & \multirow{3}{*}{0,042} \\
\hline Custo X Benefício $\leftarrow$ Estrutura Física & 0,046 & 0,466 & \\
\hline Custo X Benefício $\leftarrow$ Currículo & $-0,098$ & 0,149 & \\
\hline Relação aluno X professor $\leftarrow$ Corpo Docente & 0,414 & $0,000 *$ & \multirow{3}{*}{0,296} \\
\hline Relação aluno X professor $\leftarrow$ Estrutura Física & 0,154 & $0,005 * *$ & \\
\hline Relação aluno X professor $\leftarrow$ Currículo & 0,098 & 0,090 & \\
\hline Envolvimento $\leftarrow$ Corpo Docente & 0,109 & 0,086 & \multirow{3}{*}{0,117} \\
\hline Envolvimento $\leftarrow$ Estrutura Física & 0,155 & $0,011 * *$ & \\
\hline Envolvimento $\leftarrow$ Currículo & 0,175 & $0,007 * *$ & \\
\hline
\end{tabular}

Fonte: Dados da pesquisa. 
Ainda quanto ao curso de Administração, o corpo docente também teve impacto significativo, juntamente com a estrutura física, na relação aluno-professor, no entanto, não teve relação significativa com o envolvimento, que, por sua vez, foi precedido significativamente pela estrutura física e pelo currículo, onde os coeficientes são semelhantes.

No curso de fisioterapia (tabela 6), a relação entre aluno e professor obteve o maior coeficiente de explicação, tendo $36 \%$ das suas variações explicadas, porém a única relação significativa deste construto foi com o corpo docente. A satisfação teve o corpo docente e a estrutura física como preditores significativos. Já o custo-benefício não mostrou ter relações significativas com os atributos analisados. E por fim, o envolvimento do aluno com o curso foi significativamente precedido pelo currículo do curso.

Tabela 6 Regressão múltipla entre atributos e indicadores de resultado - curso de fisioterapia

\begin{tabular}{|c|c|c|c|}
\hline Regressão & Coeficientes $\beta$ & Sig. & $\mathbf{R}^{2}$ \\
\hline Satisfação $\leftarrow$ Corpo Docente & 0,249 & $0,003 * *$ & \multirow{3}{*}{0,189} \\
\hline Satisfação $\leftarrow$ Estrutura Física & 0,223 & $0,004 * *$ & \\
\hline Satisfação $\leftarrow$ Currículo & 0,104 & 0,197 & \\
\hline Custo X Benefício $\leftarrow$ Corpo Docente & $-0,020$ & 0,827 & \multirow{3}{*}{0,028} \\
\hline Custo X Benefício $\longleftarrow$ Estrutura Física & $-0,074$ & 0,377 & \\
\hline Custo X Benefício $\leftarrow$ Currículo & $-0,125$ & 0,157 & \\
\hline Relação aluno X professor $\leftarrow$ Corpo Docente & 0,540 & $0,000^{*}$ & \multirow{3}{*}{0,363} \\
\hline Relação aluno X professor $\leftarrow$ Estrutura Física & 0,093 & 0,169 & \\
\hline Relação aluno X professor $\leftarrow$ Currículo & 0,056 & 0,433 & \\
\hline Envolvimento $\leftarrow$ Corpo Docente & 0,065 & 0,457 & \multirow{3}{*}{0,099} \\
\hline Envolvimento $\leftarrow$ Estrutura Física & $-0,095$ & 0,238 & \\
\hline Envolvimento $\leftarrow$ Currículo & 0,288 & $0,001 *$ & \\
\hline
\end{tabular}

\footnotetext{
$* \mathrm{p} \leq 0,001 * * \mathrm{p} \leq 0,05$

Fonte: Dados da pesquisa.
}

Os dados de cada curso, agregados, geraram os resultados gerais apresentados na tabela 7, que podem ser considerados relativos a um curso de graduação. Esta agregação, denominada de amostra geral, ou seja, de todos os quatro cursos, o relacionamento entre aluno e professor teve $50 \%$ de suas variações explicadas pelos atributos, sendo precedido mais fortemente pelo corpo docente e pelo currículo. A satisfação, por sua vez, teve um relacionamento mais forte com o corpo docente, enquanto a relação entre o custo e o benefício é mais fortemente precedida pelo currículo. O envolvimento do aluno mostrou relações significativas com os três atributos, mas foi precedido com maior magnitude do coeficiente de regressão pelo corpo docente e pelo currículo. 
Tabela 7 Regressão múltipla entre atributos e indicadores de resultado - dados totais

\begin{tabular}{|c|c|c|c|}
\hline Regressão & Coeficientes $\beta$ & Sig. & $\mathbf{R}^{2}$ \\
\hline Satisfação $\leftarrow$ Corpo Docente & 0,350 & $0,000^{*}$ & \multirow{3}{*}{0,318} \\
\hline Satisfação $\leftarrow$ Estrutura Física & 0,154 & $0,000^{*}$ & \\
\hline Satisfação $\leftarrow$ Currículo & 0,165 & $0,000^{*}$ & \\
\hline Custo X Benefício $\leftarrow$ Corpo Docente & 0,085 & $0,020 * *$ & \multirow{3}{*}{0,263} \\
\hline Custo X Benefício $\leftarrow$ Estrutura Física & 0,051 & 0,114 & \\
\hline Custo X Benefício $\leftarrow$ Currículo & 0,427 & $0,000^{*}$ & \\
\hline Relação aluno X professor $\leftarrow$ Corpo Docente & 0,395 & $0,000 *$ & \multirow{3}{*}{0,506} \\
\hline Relação aluno X professor $\leftarrow$ Estrutura Física & 0,081 & $0,002 * *$ & \\
\hline Relação aluno X professor $\leftarrow$ Currículo & 0,343 & $0,000 *$ & \\
\hline Envolvimento $\leftarrow$ Corpo Docente & 0,142 & $0,000^{*}$ & \multirow{3}{*}{0,119} \\
\hline Envolvimento $\leftarrow$ Estrutura Física & 0,074 & $0,035 * *$ & \\
\hline Envolvimento $\leftarrow$ Currículo & 0,190 & $0,000^{*}$ & \\
\hline
\end{tabular}

$$
* \mathrm{p} \leq 0,001 * * \mathrm{p} \leq 0,05
$$

Fonte: Dados da pesquisa.

Na tabela 8 é apresentado um resumo dos coeficientes de explicação para cada curso, permitindo assim uma comparação horizontal entre os cursos, deste modo, a satisfação, teve o mais alto coeficiente de explicação em três das cinco análises (direito; biologia; administração) e demonstrou relações mais fortes com o atributo corpo docente nos cursos de administração e direito, enquanto a estrutura física teve participações significativas nos cursos de biologia e fisioterapia.

Tabela 8 Síntese dos coeficientes de explicação $\left(\mathrm{R}^{2}\right)$

\begin{tabular}{|c|c|c|c|c|c|}
\hline \multirow{2}{*}{ Regressão } & \multicolumn{5}{|c|}{ Coeficientes de explicação $\left(\mathbf{R}^{2}\right)$} \\
\hline & Direito & Biologia & Administração & Fisioterapia & Total \\
\hline Satisfação $\leftarrow$ Atributos & 0,320 & 0,189 & 0,291 & 0,189 & 0,318 \\
\hline Custo X Benefício $\leftarrow$ Atributos & 0,068 & 0,009 & 0,042 & 0,028 & 0,263 \\
\hline Relação aluno X professor $\leftarrow$ Atributos & 0,224 & 0,072 & 0,296 & 0,363 & 0,506 \\
\hline Envolvimento $\leftarrow$ Atributos & 0,059 & 0,095 & 0,117 & 0,099 & 0,119 \\
\hline
\end{tabular}

Fonte: Dados da pesquisa.

A análise da tabela 8 pode remeter a participação e importância de laboratórios e equipamentos para o processo de aprendizagem nos cursos de biologia e fisioterapia, enquanto nos cursos de direito e administração, o sucesso do processo e a satisfação parecem depender mais da competência do professor.

A relação entre o custo e o benefício apresentou baixos coeficientes de explicação em quatro das cinco análises feitas, uma vez que o coeficiente mais alto apresentado foi para a amostra total. Esta relação foi significativamente precedida, embora com fracos coeficientes, pelo corpo docente, que mostrou ter uma relação negativa, ou seja, ao passo que quanto maior a qualidade do corpo docente, menor é a percepção da relação entre custo-benefício 
A relação entre aluno e professor obteve coeficientes de explicação com magnitude aceitável em quatro das cinco análises (direito; administração; fisioterapia; amostra total), nessas, significativamente precedida pelo corpo docente, que parece ter uma forte participação na construção do relacionamento entre as partes. Convém ressaltar que este foi o construto com maior nível de explicação na amostra total (50\%).

O envolvimento do aluno com o curso parece aumentar quando há um aumento na qualidade do currículo e da estrutura física, variáveis as quais demonstraram coeficientes de regressão com maiores magnitudes.

Os três atributos em análise (corpo docente; estrutura física; currículo) tem sua importância para a construção da oferta de cursos de graduação muito difundida pela literatura (KOTLER; FOX, 1994), no entanto este estudo, corroborando com esta importância, conduz a um entendimento do impacto destas variáveis em indicadores de resultado para a IES pesquisada, o que pode auxiliar no planejamento e ações do curso de forma orientada pelo mercado, uma vez que torna possível mensurar o impacto que determinada ação poderá ter em indicadores de desempenho.

\section{CONCLUSÕES E IMPLICAÇÕES}

No estudo apresentado foram identificados os principais atributos a serem contemplados em diferentes cursos de graduação, destacando-se o corpo docente, a estrutura física e o currículo. Verificou-se através da análise dos dados a importância de um curso estar próximo das demandas do mercado no qual a instituição está inserida, apoiado por um currículo, corpo docente e estrutura física que aproxime a oferta "intangível” da demanda.

Conforme apresentados, os atributos tornam-se importantes indicadores de direção para nortear as atividades de gestão de um curso de forma a focar um melhor atendimento aos seus stakeholders, uma vez que essa abordagem torna-se importante para melhor atender as expectativas e desejos dos stakeholders da instituição (ABRAHAMSON, 2000). Os atributos nesse sentido auxiliam em um maior direcionamento nas atividades de ensino, pesquisa e extensão, uma vez que trazem itens pontuais quanto a idealização de um corpo docente, uma estrutura física, um currículo, bases essas que empiricamente e teoricamente constituem o escopo de um curso de graduação (KOTLER; FOX, 1994).

Os resultados evidenciaram que os atributos explorados junto aos stakeholders da IES possuem relações significativas com indicadores de desempenho de um curso de graduação, analisados pelos mesmos stakeholders. Nisto direcionar a oferta dos cursos de graduação, 
seguindo os preceitos da orientação para mercado e procurando prospectar os resultados das ações de orientação é possível e, ademais, é necessário em tempos competitivos no setor de ensino.

Gerencialmente, a partir desse tipo de pesquisa, os gestores podem posicionar os cursos de acordo com suas peculiaridades de mercado, bem como a IES identificar os atributos e indicadores de performance que são o eixo do posicionamento de sua marca, visto que os indicadores gerais indicam o caminho para a avaliação e ou elaboração do posicionamento institucional.

O método utilizado demonstrou-se eficiente para alcançar o objetivo proposto, sendo o mesmo de fácil operacionalização. Tal método pode ser utilizado em estudos futuros em outros contextos, instituições e cursos, auxiliando dessa forma os gestores de IES a adotarem posturas mais pró-ativas e orientadas para o mercado. A abordagem em duas etapas (exploratória e descritiva) é importante para verificar nuanças que possam ter em virtude dos diferentes contextos pesquisados, porém como a pesquisa evidenciou, há atributos e indicadores que são comuns a diferentes cursos de graduação. A coleta dos dados tanto através de entrevistas em profundidade, quanto de grupos de foco foi eficiente para os propósitos da etapa exploratória, sendo assim recomendadas para este tipo de estudo no contexto de ensino superior. Convém ressaltar a importância, na análise de dados qualitativos, da presença de dois ou mais pesquisadores, para que seja possível comparar as categorias geradas entre os pesquisadores, reduzindo assim os possíveis vieses de interpretação na análise de conteúdo.

Já na etapa descritiva, a forma de amostra mostrou-se adequada para generalizações de resultados neste contexto estudado, uma vez que com bancos de dados disponíveis, técnicas probabilísticas devem ser utilizadas a garantir uma imparcialidade e prover a todos os entrevistados a mesma probabilidade de participar do estudo. Outro fator relevante na amostra é condizente com o cálculo do tamanho amostral, que permite realizar inferências sobre a população. Todavia deve-se atentar para que os tamanhos amostrais também garantam a utilização das técnicas estatísticas preconizadas. Na análise da etapa descritiva é importante o uso de técnicas multivariadas que possibilitem a análise das relações, sendo possível identificar efeitos de possíveis ações nos atributos. A técnica de regressão utilizada se mostrou eficiente para isto.

Ainda em relação ao método, ao auxiliar na identificação de atributos que traduzam as expectativas e desejos dos stakeholders, permite a elaboração de estratégias e ações de gestão, 
ensino, pesquisa e extensão, que podem refletir numa maior capacidade competitiva dos cursos e das IES.

Em estudos futuros é indicado que seja pesquisado os alunos evadidos do curso, o que permitiria analisar suas razões em comparação com as informações obtidas junto aos outros stakeholders definidos. Ainda, os egressos, que nesta pesquisa foram incluídos no universo de profissionais podem ser estratificados e analisados de forma comparativa com os egressos de outras faculdades, situação essa que permitiria uma avaliação da concorrência.

Cabe ressaltar que a replicação do estudo em cursos de ensino superior com características distintas pode, além de solidificar os atributos e propiciar o surgimento de indicadores consistentes, corroborar com os resultados encontrados que comprovam a aplicabilidade prática da metodologia para o entendimento das percepções dos diversos stakeholders envolvidos com uma IES.

A priori as limitações desse estudo referem-se as suas generalizações dos resultados visto que foi realizado em um mercado, determinados cursos em uma única instituição, contudo o método pode ser aplicado e validado em outros contextos. Todavia as generalizações podem ser feitas para as populações em estudo, visto a técnica de amostragem utilizada na etapa descritiva-quantitativa.

Finalmente destaca-se a importância da pesquisa de marketing para o desenvolvimento de cursos de graduação mais orientados para o mercado, gerando dessa forma indicadores que norteiam a gestão, o ensino, a pesquisa e a extensão, através do entendimento das expectativas de diferentes stakeholders, proporcionando serviços de maior qualidade e ao mesmo tempo auxiliando na geração de processos criativos e inovadores que impliquem na criação de vantagens competitivas a esse produto e às organizações que o ofertam.

\section{REFERÊNCIAS}

ABRAHAMSON, T. Life and death on the Internet: To web or not to web is no longer a question. Journal of College Admission, v. 168, p. 6-11, 2000.

ANTONI, V. L. A Relação entre Orientação para o Mercado e Performance

Organizacional: um Estudo nos Cursos de Bacharelado em Administração da Região Sul do Brasil. 2004. Tese (Doutorado em Engenharia de Produção) - PPGEP, UFSC, Florianópolis, 2004.

ANTONI, V. L.; DAMACENA, C.; LEZANA, Á. G. R. Um Modelo Preditivo de Orientação para o Mercado: um Estudo no Contexto do Ensino Superior Brasileiro. In: ENANPAD, 28, 2006, Curitiba (PR). Anais... Rio de Janeiro: ANPAD, 2004. 
BARDIN, L. Análise de Conteúdo. 3. ed. Lisboa: Edições 70, 2004.

CAPPELle, M. C. A.; MELO, M. C. O. L.; GONÇALVES, C. A. Análise de conteúdo e análise de discurso nas ciências sociais. Organizações Rurais \& Agroindustriais, v. 5, n. 1, p. 1-15, 2003.

DAY, G. S. The Capabilities of Market-Driven Organizations. Journal of Marketing, v. 58 , n. 4, p. 37-52, 1994.

DURVASULA, S.; LYSONSKI, S.; MEHTA, S. C. Business-to-business marketing Service recovery and customer satisfaction issues with ocean shipping lines. European Journal of Marketing, v. 34, n. 3, p. 433-452, 2000.

EAGLE, L.; BRENNAN, R. Are students customers? TQM and marketing perspectives. Journal of Quality Assurance in Education, v. 15, n. 1, p. 44-60, 2007.

EBOLI, M. Educação e modernidade nas organizações: desafio de implantar sistemas educacionais competitivos. In: Educação para o século XXI, São Paulo: Schmukler, 1999. p. 61 - 75. (Coletânea Universidades Coorporativas).

FALEIRO, S. N. A relação entre orientação para o mercado, orientação para a aprendizagem e inovação. $O$ caso dos cursos de graduação em administração filiados a ANGRAD. 2001. Dissertação (Mestrado em Administração) - PPGA / Escola de Administração, UFRGS, Porto Alegre, 2001.

FROEMMING, L. M. S. Encontros de serviços em uma instituição de ensino superior. 2001. Tese (Doutorado em Administração) - PPGA / Escola de Administração, UFRGS, Porto Alegre, 2001.

GALOTTI, K. M.; MARK, M. C. How do high school students structure an important life decision? A short-term longitudinal study of the college decision-making process. Research in Higher Education, v. 35, n. 5, p. 589-607, 1994.

GUSTAFSSON, A.; JOHNSON, M. D. Determining Attribute Importance in a Service Satisfaction Model. Journal of Service Research, v. 7, n. 2, p. 124-142, 2004.

HAIR, J. F.; BABIN, B.; MONEY, A. H.; SAMOUEL, P. Fundamentos de Métodos de Pesquisa em Administração. Porto Alegre: Bookman, 2005.

HU, S.; HOSSLER, D. Willingness to pay and preference for private institutions. Research in Higher Education, v. 41, n. 6, p. 685-701, 2000.

INEP - INSTITUTO NACIONAL DE ESTUDOS E PESQUISAS EDUCACIONAIS. Página institucional. Disponível em: <http://www.inep.gov.br>. Acesso em: Abr. 2012.

JAAKKOLA, E. Purchase decision-making within professional consumer services: Organizational or consumer buying behavior? Marketing Theory, v. 7, n. 1, p. 93-108, 2007. 
JAWORSKI, B. J.; KOHLI, A. K. Market Orientation: Antecedents and Consequences. Journal of Marketing, v. 57, n. 3, p. 53-70, 1993.

KELLEY, S. W.; DONNELLY, J. H.; SKINNER, S. J. Customer participation in service production and delivery. Journal of Retailing, v. 66, n. 3, p. 315-350, 1990.

KOHLI, A. K.; JAWORSKI, B. J. Market orientation: the construct, research propositions, and managerial applications. Journal of Marketing, v. 54, n. 2, p. 1-18, 1990.

KOTLER, P.; FOX, K. F. Marketing estratégico para instituições educacionais. São Paulo: Atlas, 1994.

LEITE, R. S.; LOPES, H. E. G.; LEITE, D. S. A Qualidade Percebida no Ensino Superior: Um Estudo em uma Instituição do Centro-Oeste de Minas Gerais. In: ENANPAD, 30, 2006, Salvador (BA). Anais... Rio de Janeiro: ANPAD, 2006.

LOVELOCK, C.; WRIGHT, L. Serviços: marketing e gestão. São Paulo: Saraiva, 2003.

MALHOTRA, N. K. Pesquisa de Marketing: uma orientação aplicada. 4. ed. Porto Alegre: Bookman, 2006.

MARINGE, F. Implications for positioning, recruitment and marketing. International Journal of Educational Management, v. 20, n. 6, p. 466-479, 2006.

MINAYO, M. C. S.; SANCHES, O. Quantitativo-Qualitativo: Oposição ou complementaridade? Caderno de Saúde Pública, v. 9, n. 3, p.239-248, 1993.

NARVER, J. C.; SLATER, S. F. The Effect of Market Orientation on Business Profitability. Journal of Marketing, v. 54, n. 4, p. 20-35, 1990.

NAUDÉ, P.; IVY, J. The marketing strategies of universities in the United Kingdom. The International Journal of Educational Management, v. 13, n. 3, p. 126-134, 1999.

NEVES, J. L. Pesquisa Qualitativa - Características, Usos e Potencialidades. Caderno de Pesquisas em Administração, v. 1, n. 3, p.1-5, 1996.

NICOLAO, L.; ESPARTEL, L. B. Julgamentos pós-escolha do curso de administração: o papel da qualidade percebida, da satisfação e do arrependimento e os seus comportamentos subseqüentes. In: ENANPAD, 28, 2006, Curitiba (PR). Anais... Rio de Janeiro: ANPAD, 2004.

NILSSON-WITELL, L.; FUNDIN, A. Dynamics of service attributes: a test of Kano's theory of attractive quality. International Journal of Service Industry Management, v. 16, n. 2 ; p. 152-169, 2005.

ODIN, J. K.; MANICAS, P. T. Globalization and Higher Education. Hawai: University of Hawaii Press, 2004. 
OLIVEIRA, M.; FREITAS, H. M. R. Focus group, pesquisa qualitativa: resgatando a teoria instrumentalizando o seu planejamento. Revista de Administração da USP, v. 33, n. 3. p. 83$91,1998$.

SEVIER, R. A. Recruiting African-American undergraduates: A national survey of the factors that affect institutional choice. College and University, v. 68, n. 1, p. 48-52, 1993.

SIMPSON, P. M.; SIGUAW, J. A. Student Evaluation of teaching: an exploratory study of the faculty response. Journal of Marketing Education, v. 22, n.3, p. 199-213, 2000.

SIQUEIRA, R. P.; CARVALHO, J. L. F. Qualidade do Serviço Educacional Prestado por Escolas de Administração: Confronto Entre uma Universidade Pública e uma Faculdade Privada. In: ENANPAD, 30, 2006, Salvador (BA). Anais... Rio de Janeiro: ANPAD, 2006.

SOUZA, E. C. L.; SOUZA, C. C. L.; ASSIS, S. A. G.; ZERBINI, T. Métodos e Técnicas de Ensino e Recursos Didáticos para o Ensino do Empreendedorismo em IES Brasileiras. In: ENANPAD, 28, 2006, Curitiba (PR). Anais... Rio de Janeiro: ANPAD, 2004.

SPIES, R. R. The effect of rising costs on college choice: A study of the application decisions of high-ability students. College Entrance Examination Board. Nova York, 1978.

TACHIZAWA, T.; ANDRADE, R. O. B. Gestão de instituições de ensino. 2 ed. Rio de Janeiro: Editora FGV, 2001.

VENKATESH, U. The Importance of Managing Points-of-Marketing in Marketing Higher Education Programmes - Some Conclusions. Journal of Services Research, v. 1, n. 1, p. 125-141, 2001.

ZEITHAML, V. A.; BITNER, M. J. Services Marketing. Singapura: McGraw-Hill, 1996. 